\title{
HISTOPATHOLOGICAL CHANGES OF FALLOPIAN TUBE IN UTERINE MALIGNANCIES
}

\author{
Arsha V1, Lillykutty Pothen'2, S. Sankar ${ }^{3}$
}

1 Postgraduate Student, Department of Pathology, Government Medical College, Kottayam, Kerala. ${ }^{2}$ Associate Professor, Department of Pathology, Government Medical College, Kottayam, Kerala. 3 Professor and HOD, Department of Pathology, Government Medical College, Kottayam, Kerala.

\section{BACKGROUND}

ABSTRACT

Malignant neoplasms of uterus account for significant proportion of all female cancers, though rare in India compared to Western countries. Extrauterine involvement has a significant effect on prognosis and treatment decision. Hence, thorough sampling of uterus and adnexal structures is important for appropriate staging. Fallopian tube is not only an origin of ovarian serous carcinoma, but also an important passage for endometriosis and also has an indirect role in pathogenesis of endometrioid carcinoma, serous carcinoma and clear cell carcinoma. According to studies, fimbrial ends of fallopian tubes show more chances of lesions than the usual sampling sites like middle portion and base in classical method.

The objectives of the study were to describe the histopathological changes of fallopian tube associated with uterine malignancies and to describe the findings of fallopian tube at the base, middle portion and fimbria in uterine malignancies.

\section{MATERIALS AND METHODS}

A descriptive study was conducted on specimens of all the cases of total abdominal hysterectomy and bilateral salpingooophorectomy diagnosed with uterine malignancies received in Department of Pathology, Govt. Medical College, Kottayam during the study period (May 2016 - May 2017).

\section{RESULTS}

40 cases of uterine malignancies were studied for fallopian tube changes, of which 15 cases showed histopathological changes in fallopian tube. Of these 7 cases (46.7\%) showed changes in the fimbria, 5 cases (33.3\%) showed changes in the mid portion and fimbriae simultaneously, 2 cases (13.3\%) showed changes in the base and one case (6.7\%) showed changes in the middle portion alone. The histopathological changes were more frequent in fimbria compared to middle portion and base of fallopian tube.

\section{CONCLUSION}

The changes observed in fallopian tube were endometriosis, dysplasia, carcinoma, inflammation and salpingitis isthmica nodosa. The changes were more frequent in fimbria compared to middle portion and base of fallopian tube.

\section{KEYWORDS}

Fallopian Tube; Endometrial Carcinoma; Dysplasia.

HOW TO CITE THIS ARTICLE: Arsha V, Pothen L, Sankar S. Histopathological changes of fallopian tube in uterine malignancies. J. Evolution Med. Dent. Sci. 2018;7(14):1786-1791, DOI: 10.14260/jemds/2018/403

\section{BACKGROUND}

The malignant neoplasms of uterus account for a significant proportion of all female cancers. Even though cervical carcinoma was more prevalent than endometrial carcinoma worldwide, earlier detection through cervical cytology and eradication of squamous intraepithelial lesions in the cervix has decreased its occurrence. The most common carcinoma of uterine corpus is endometrial carcinoma.

Endometrial carcinoma usually arises in postmenopausal women. More than $80 \%$ of endometrial carcinoma is endometrioid type. In addition to the endometrioid carcinoma, special variants of uterine corpus malignancies are uterine serous carcinoma, clear cell carcinoma, pure squamous cell carcinoma, mixed carcinoma and undifferentiated carcinoma.

'Financial or Other Competing Interest': None.

Submission 19-01-2018, Peer Review 14-03-2018,

Acceptance 21-03-2018, Published 02-04-2018.

Corresponding Author:

Dr. Lillykutty Pothen,

Department of Pathology,

Government Medical College,

Kottayam-686008, Kerala.

E-mail: gkaniankunnel@yahoo.com

DOI: $10.14260 /$ jemds $/ 2018 / 403$
The common malignant mesenchymal tumours of uterus include leiomyosarcoma, endometrial stromal sarcoma and undifferentiated uterine sarcoma.

FIGO staging of endometrial carcinoma include those confined to uterine corpus under stage I, uterine cervix when involved stage II and stage III includes those with pelvic extension that is invasion to adnexae and stage IV is extrapelvic extension. Extrauterine involvement has a significant effect on prognosis and treatment decision. Hence, thorough sampling of uterus and adnexal structures is very important for appropriate staging.

Fallopian tube is found to have significant role in the origin of ovarian serous carcinoma. Similarly, studies have shown that fallopian tube changes are commonly seen associated with serous carcinoma uterus. Also, it is an important passage for endometriosis. Endometrioid endometrial carcinomas have reports of simultaneous occurrence of endometrioid type cancers in fallopian tube. This signifies the thorough sampling of adnexae in case of uterine malignancies.

According to many studies, fimbrial ends of fallopian tubes show more chances of lesions than the usual sampling sites in classical method. ${ }^{1}$ Indian studies based on these areas are limited. In the classical method, sections are usually taken from middle portion of fallopian tubes. A new 
protocol known as the SEE-FIM (Sectioning and Extensively Examining the Fimbriated End of Fallopian Tube) protocol is found to be more useful than the classical method, as it includes sectioning the fimbrial portion at $2 \mathrm{~mm}$ intervals in longitudinal fashion and rest of the fallopian tube in crosssectional pattern. ${ }^{2}$

This study was carried out to describe the fallopian tube lesions in uterine malignancies and to describe the findings in fallopian tube at the base, middle portion and fimbria in uterine malignancies.

\section{MATERIALS AND METHODS}

A descriptive study was conducted on specimens of all the cases of total abdominal hysterectomy and bilateral salpingooophorectomy diagnosed with uterine malignancies received in Department of Pathology, Govt. Medical College, Kottayam during the study period (May 2016 - May 2017).

\section{Inclusion Criteria}

Histopathologically, diagnosed cases of uterine malignancies (all the morphological subtypes- Endometrioid endometrial carcinoma being the most common) were included.

\section{Exclusion Criteria}

Total abdominal hysterectomy and bilateral salpingooophorectomy specimens in which surgeries done for ovarian tumours cases with indefinite diagnosis etc. were excluded.

\section{Sample Size}

According to previous statistics, the total number of specimens of uterine malignancies received in the Department of Pathology, Govt. Medical College, Kottayam per year was approximately 40 - 50. A pilot study was done with samples received in the department during a period of one month and got 2 cases of endometrial carcinoma. One of them showed focal dysplasia in the fallopian tube. Hence, we studied all the specimens of uterine malignancies received in the Department of Pathology, Govt. Medical College, Kottayam during the study period.

\section{Study Procedure}

Clinical details of each case were recorded initially. Then examination of the gross specimen was done. Appropriate bits of tissues from representative areas to be studied were taken. Sampling of fallopian tube was done according to SEE-FIM protocol, that is longitudinal sections were taken from the fimbria and cross-sections from rest of the fallopian tube including middle portion and base. All specimens were fixed in formalin and embedded in paraffin. 4 microns thick sections were stained with $\mathrm{H}$ and $\mathrm{E}$ for routine histological examination.

\section{Data Management and Analysis}

The data were entered in Microsoft Excel and further statistical analysis was done using SPSS software. Ratio, proportion and frequency were used.

\section{RESULTS}

A total of 40 cases, which were satisfying the inclusion criteria were analysed. The following are the results obtained.

\section{Age Distribution of Cases}

Among the 40 cases of uterine malignancies, 16 cases (40\%) were presented in 51 - 60 years' age group, 10 cases (25\%) in 41 - 50 years' age group, 8 cases (20\%) in $61-70$ years, 4 cases $(10 \%)$ were in 71 - 80 years and 2 cases (5\%) were in $31-40$ years' age group.

\section{Clinical Features}

The clinical presentation of 25 cases (62\%) was postmenopausal bleeding. 9 cases (22\%) presented with menorrhagia. 3 cases (8\%) with pain abdomen. The clinical presentation of 2 cases (5\%) was mass abdomen and one case $(3 \%)$ was bloody discharge per vaginum.

\section{Gross Pathology}

Grossly, the neoplasm was infiltrating less than half of myometrium in 22 cases (55\%) and more than half of myometrium in 17 cases (42\%). The myometrial invasion was not applicable in one case (3\%), which was sarcoma.

\section{Distribution of Histological Types of Uterine Corpus Malignancies}

Among the 40 cases of uterine corpus malignancies, 27 cases (67\%) were Endometrioid endometrial carcinoma, 5 cases $(12 \%)$ were Serous carcinoma, 4 cases $(10 \%)$ were Carcinosarcoma, 3 cases (8\%) were Clear cell carcinoma and one case (3\%) was Endometrial stromal sarcoma.

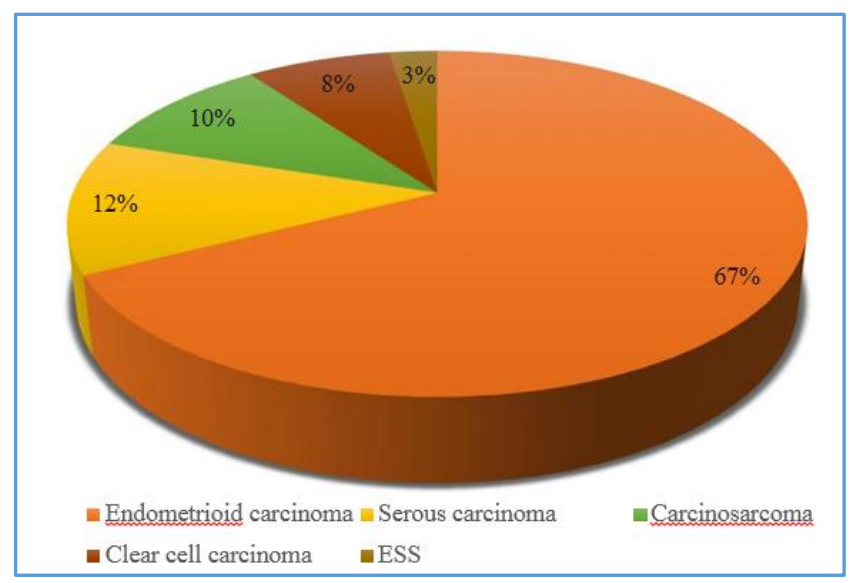

\section{Figure 1. Pie Diagram showing Distribution of Histopathological Types of Uterine Corpus Malignancies}

\section{FIGO Staging of Uterine Corpus Malignancies}

Of the 40 cases, 29 cases (72.5\%) presented in Stage I. Five cases $(12.5 \%)$ presented in Stage II. Another five cases $(12.5 \%)$ were in Stage III and one case $(2.5 \%)$ presented in Stage IV.

\section{Histopathological Changes of Fallopian Tube}

The fallopian tubes of the 40 cases studied showed various histopathological changes, both non-neoplastic and neoplastic. The changes were seen in 15 cases (37.5\%). 25 cases $(62.5 \%)$ showed no significant change in fallopian tube histology.

Distribution of types of Histopathological Changes of Fallopian Tube

Out of the 15 cases of uterine malignancies associated with 
fallopian tube changes, 6 cases $(40 \%)$ were showing nonneoplastic changes and 9 cases $(60 \%)$ were showing dysplasia and neoplastic changes.

\begin{tabular}{|c|c|c|}
\hline Fallopian Tube Changes & Frequency & Percentage \\
\hline Non-neoplastic changes & 6 & 40 \\
\hline $\begin{array}{c}\text { Dysplastic and Neoplastic } \\
\text { changes }\end{array}$ & 9 & 60 \\
\hline $\begin{array}{c}\text { Table 1. Frequency and Percentage of Neoplastic and } \\
\text { Non-Neoplastic Changes in Fallopian Tube }\end{array}$ \\
\hline
\end{tabular}

The fallopian tube changes were endometriosis in one case (2\%), dysplasia in 4 cases $(10 \%)$ and carcinoma in 2 cases (5\%). 3 cases (8\%) showed carcinoma on one fallopian tube with dysplasia on other side. Inflammation was seen in 3 cases (7\%), salpingitis isthmica nodosa in 2 cases (5\%) and there was no change in fallopian tube in the remaining 25 cases $(63 \%)$.

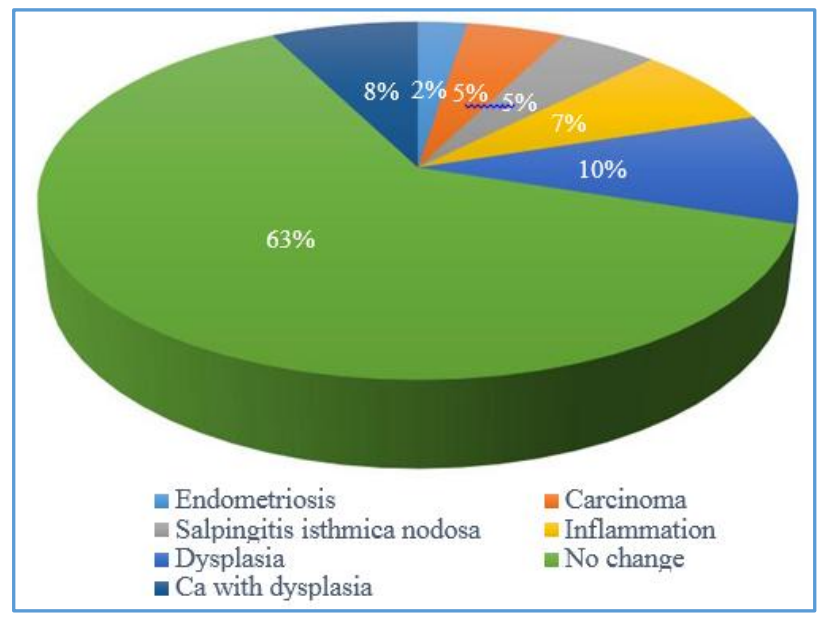

Figure 2. Pie Diagram showing Distribution of Histopathological Changes of Fallopian Tube

Out of the 27 endometrioid carcinoma cases studied, one case $(4 \%)$ showed endometriosis in fallopian tube, 3 cases (11\%) showed dysplasia, 2 cases (7\%) showed carcinoma and one case $(4 \%)$ showed carcinoma on one fallopian tube with dysplasia on the other tube. Inflammation was seen in 2 cases $(7 \%)$.

The fimbriae showed endometriosis, dysplasia, carcinoma, inflammation and salpingitis isthmica nodosa. The middle portion showed carcinoma, dysplasia and inflammation in various cases. Base of the tube showed carcinoma infiltration. There was no change in fallopian tube in the remaining 18 cases (67\%) of endometrioid carcinomas.

Out of 5 cases of serous carcinoma endometrium, 2 cases (40\%) showed carcinoma in one fallopian tube and dysplasia on other fallopian tube. The fimbriae and the middle portion simultaneously showed these changes in both cases. The base showed no change. The other 3 cases (60\%) showed no change. Out of the 4 carcinosarcoma cases, one case $(25 \%)$ showed dysplasia in the fallopian tube and that was in the fimbria and the rest 3 cases (75\%) showed no change in the tube.

Among the 3 cases of clear cell carcinoma endometrium one case (34\%) showed inflammation, the other one demonstrated salpingitis isthmica nodosa (33\%) and the third case $(33 \%)$ showed no change. The changes were found only in the fimbriae.

There was one case $(100 \%)$ of endometrial stromal sarcoma among the 40 cases studied and it showed salpingitis isthmica nodosa in middle portion of the fallopian tube.

\section{Distribution of Fallopian Tube Changes based On Site}

Of the 15 cases 7 cases (46.7\%) showed changes in the fimbria, 5 cases $(33.3 \%)$ showed changes in the mid portion and fimbriae of fallopian tube simultaneously, 2 cases $(13.3 \%)$ showed changes in the base and one case $(6.7 \%)$ showed changes in the middle portion alone.

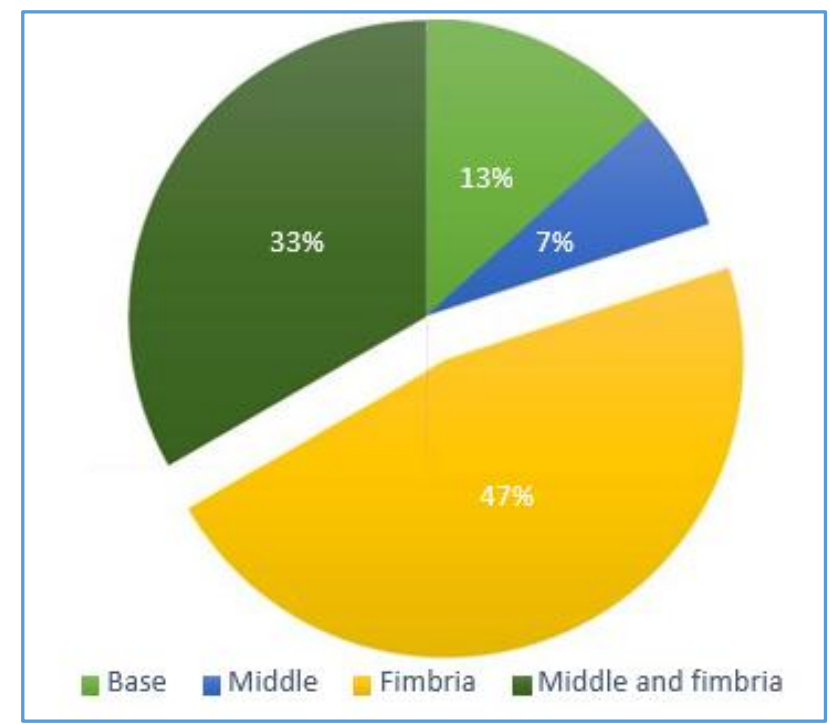

Figure 3. Pie Diagram showing Fallopian Tube Changes in Base, Middle Portion and Fimbria

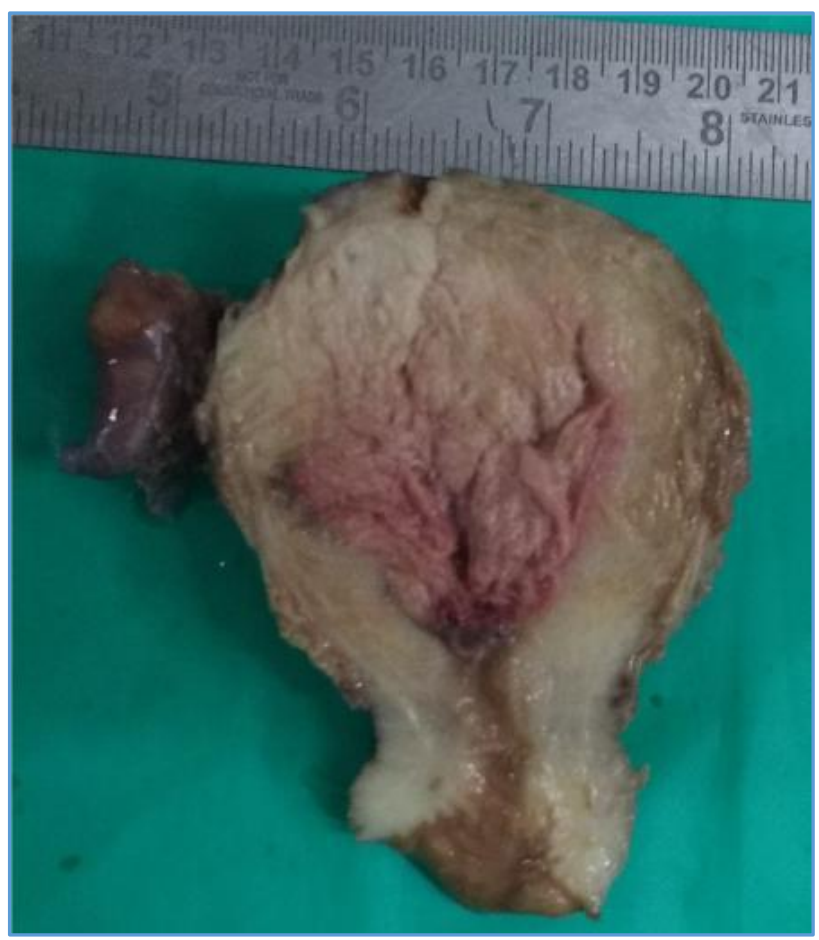

Figure 4. Gross Photograph of Serous Carcinoma Endometrium. Cut Section is Grey White, Friable 


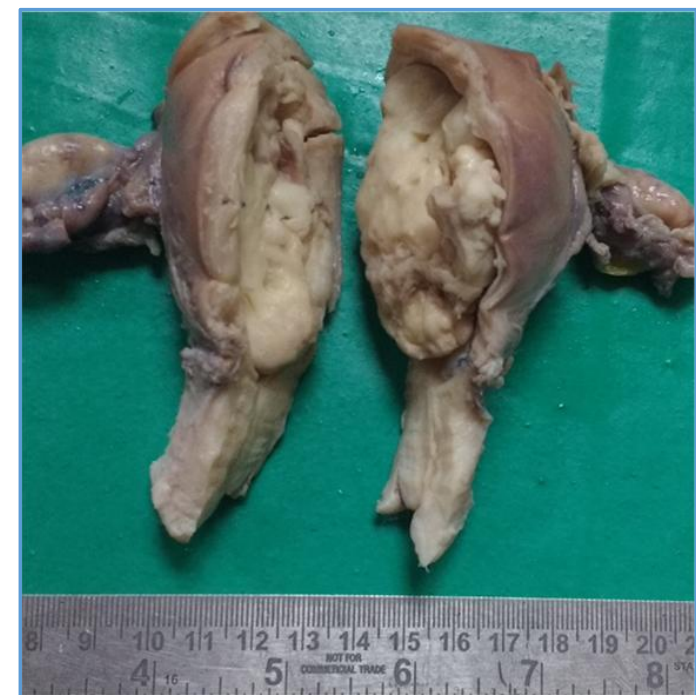

Figure 5. Gross Photograph of Carcinosarcoma Uterus. Cut Section is Grey White

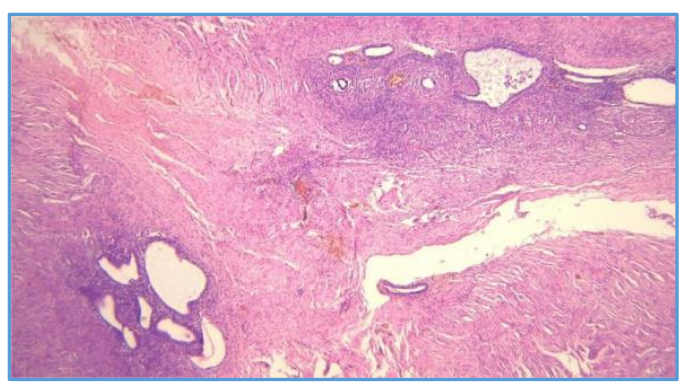

Figure 6. Photomicrograph (10x) of Endometriosis in Fallopian Tube (H and E Stain)

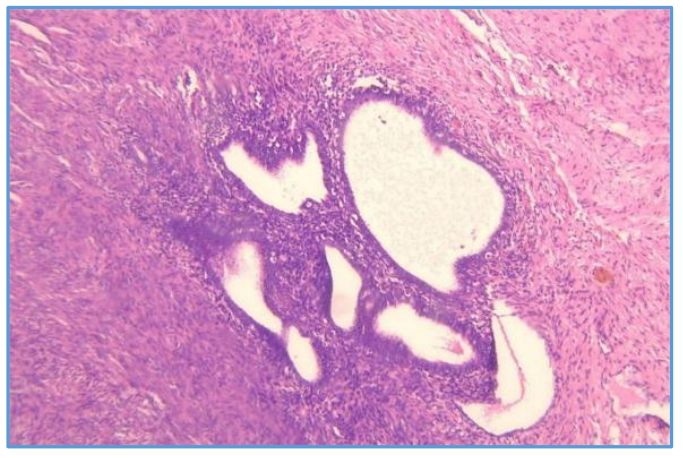

Figure 7. Photomicrograph (40x) of Endometriosis in Fallopian Tube (H and E Stain)

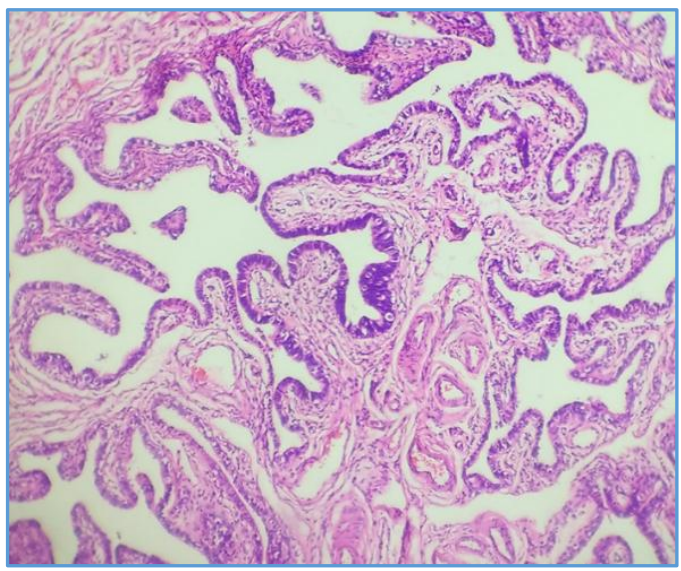

Figure 8. Photomicrograph (10x) of Dysplasia in Fallopian Tube (H and E Stain)

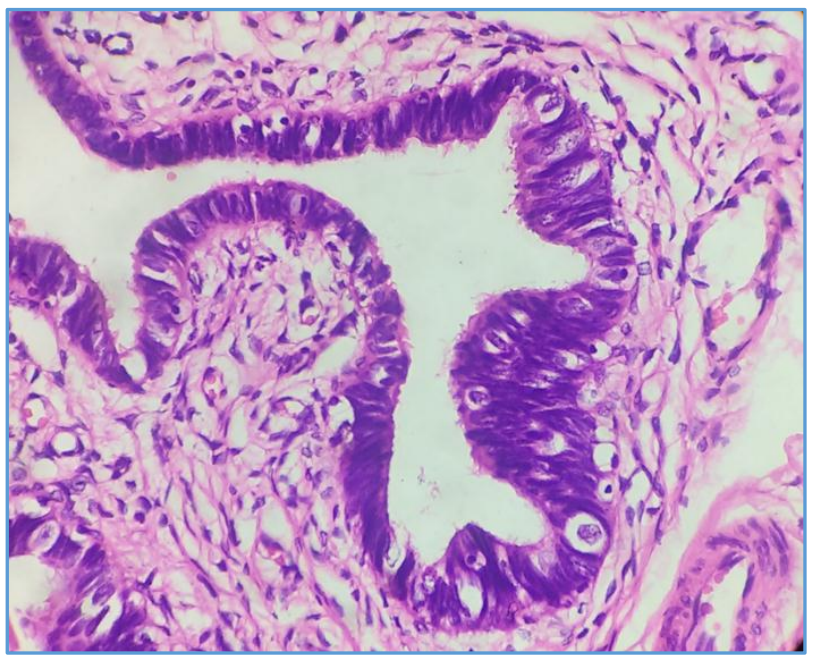

Figure 9. Photomicrograph (40x) of Dysplasia in Fallopian Tube (H and E Stain)

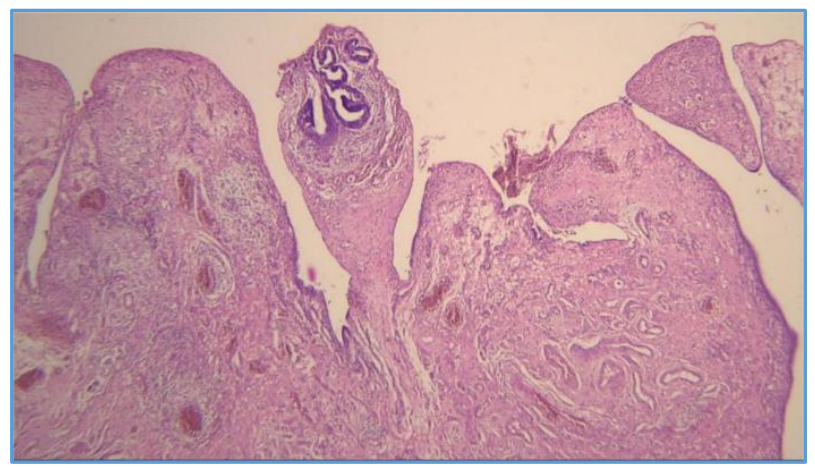

Figure 10. Photomicrograph (10x) of Carcinoma in Fallopian Tube (H and E Stain)

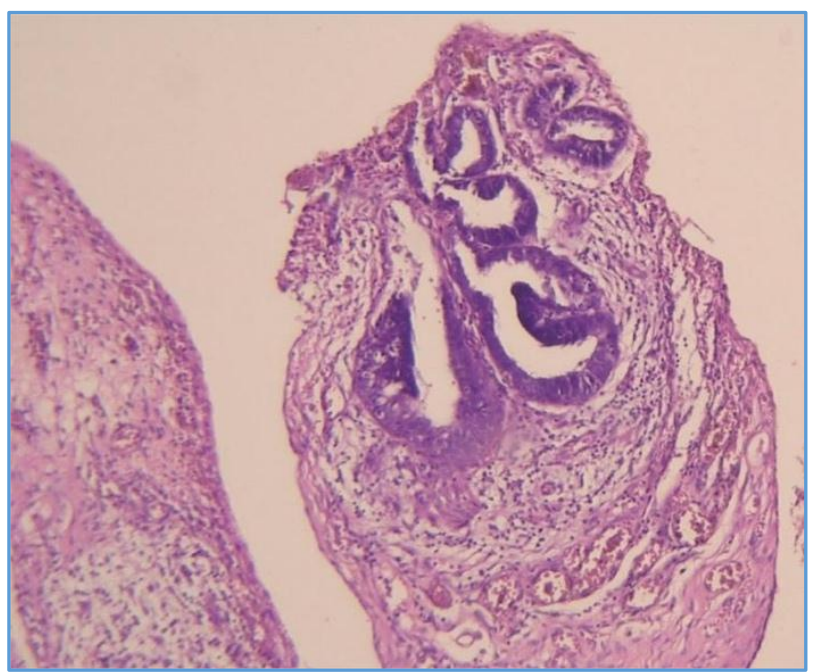

Figure 11. Photomicrograph (40x) of Carcinoma in Fallopian Tube (H and E Stain)

\section{DISCUSSION}

Endometrioid endometrial carcinoma is the most common uterine corpus malignancy. The detailed sampling and studying the pathology of uterus and adnexae is important, as it affects the staging of carcinoma.

The usual method of fallopian tube sampling is the classical method. But based on SEE-FIM protocol extensive sampling of fallopian tube, particularly the fimbria is advised. The histopathological changes are more in distal 
end of fallopian tube than proximal end. So, the chances of misdiagnoses can be reduced.

Among the carcinomas the serous carcinoma, endometrioid carcinoma, clear cell carcinoma of uterus are the commoner ones associated with changes in fallopian tube. The commonly encountered significant changes include papillary epithelial hyperplasia and other mucosal proliferations, dysplasia, atypical or adenomatous hyperplasia, endometriosis etc. Serous carcinoma of uterus can have the precursor lesions in particular like STIC, STIL, SCOUT etc. in the fallopian tube. ${ }^{3}$

Uterine serous papillary carcinoma is frequently associated with malignant epithelial changes like carcinoma in situ changes, in the remaining Mullerian epithelium. These finding suggests a transepithelial tumour spread. This type of spread is frequently seen on serosal surfaces in serous ovarian carcinoma cases. In this way, uterine serous papillary carcinoma is more similar to its ovarian counterpart. ${ }^{4}$ Theoretically, these are all favouring the concept of fallopian tube involvement associated with uterine malignancies.

Fallopian tubes are also found to be passages for endometriosis and have role in the pathogenesis of endometrioid and clear cell carcinoma. Patients with endometrioid tumours on the fimbrial ends have more aggressive clinical behaviour than that located in other parts of the fallopian tube. 5 Fimbrial lesions are more important, because they can easily spill over into the abdominal cavity.

The present study was conducted on 40 cases of uterine corpus malignancies received in Department of Pathology, Government Medical College, Kottayam. The fallopian tube was sampled in such a way that the fimbria was also extensively sampled in addition to middle portion and base.

The mean age of patients with uterine corpus malignancies in the present study was 56.3 years, which is comparable with the studies done by Ibrahim Kulac et al, ${ }^{1}$ which was 60.6 years in the specimens in which fallopian tube was studied through classical method and 58.7 years in the cases studied through new sampling method of fallopian tube. The mean age was 68.9 years in the study conducted by LB Jordan et $\mathrm{al}^{4}$ and 67.8 years in the study conducted by Fadare 0 et al. 6

The most common clinical presentation in the present study is postmenopausal vaginal bleeding (62\%) and second most common is menorrhagia (22\%). It is comparable to the following studies. The study conducted by LB Jordan et $\mathrm{al}^{4}$ showed that $80 \%$ cases presented with postmenopausal bleeding and the study by Ibrahim Kulac et $\mathrm{al}^{1}$ showed abnormal vaginal bleeding as the most common presentation. Abnormal uterine/ vaginal bleeding was the most common presentation in the study conducted by Culton LK et al. 7

In the present study, 22 cases had invasion of neoplasm upto less than half of myometrium and 17 cases had invasion to more than half of myometrium. One case was sarcoma, in which invasion was not applicable. The study conducted by Ibrahim Kulac et al $^{1}$ showed 94 cases with less than half myometrial invasion and 73 cases with more than half myometrial invasion. 33 cases were without myometrial invasion.

In the present study, 29 cases $(72.5 \%)$ of uterine malignancies were in stage I, 5 cases $(12.5 \%)$ were in stage
II and III each and one case (2.5\%) was in stage IV which is not in league with the study conducted by Fadare 0 et al, ${ }^{6}$ which showed 18 cases (36\%) in stage I, 8 cases (16\%) in stage II, 14 cases $(28 \%)$ in stage III, 9 cases $(18 \%)$ in stage IV and one case (2\%) in pT0. 9 cases (70\%) were in stage I, 3 cases $(23 \%)$ were in stage II and 1 case $(7 \%)$ was in stage III in a study conducted by Culton LK et al. ${ }^{7}$

In the present study, 15 out of 40 uterine malignancies studied showed fallopian tube changes. The changes were more in fimbriae compared to middle portion and base of the tube. Of the 15 cases 7 cases (46.7\%) showed changes in the fimbria, 5 cases $(33.3 \%)$ showed changes in the mid portion and fimbriae of fallopian tube simultaneously, 2 cases $(13.3 \%)$ showed changes in the base and one case (6.7\%) showed changes in the middle portion alone. 7 cases were having unilateral and 8 cases were having bilateral fallopian tube involvement. The changes included endometriosis, carcinoma, dysplasia, inflammation and salpingitis isthmica nodosum.

Endometriosis was seen in fimbriae and it was associated with endometrioid carcinoma, which was in league with the study conducted by Ibrahim Kulac et al. ${ }^{1}$

The study showed that there were no microscopic lesions in the fallopian tubes of cases of uterine malignancies (endometrioid carcinoma), which were sampled based on classical method (100 cases) whereas 4 cases showed changes in fallopian tube which were sampled by new method (100 cases). Of the 4 cases, fimbriae were only involved in 3 cases with unilateral involvement and infundibular-ampullary region was involved in one case which was bilateral involvement. The present study was in league with the study by Ibrahim Kulac et $\mathrm{al}^{1}$ in the fact that fimbriae are more involved compared to middle portion and base of fallopian tube.

Two of them were infiltrative carcinoma with one case in fimbria and other in infundibular-ampullary region. Other two were in fimbriae, which were proliferative glandular lesion. The infundibular region with infiltrative carcinoma was having associated endometriosis. One of the fimbriae with proliferative lesion was also having endometriosis. The study conducted by Tolcher et $\mathrm{al}^{8}$ showed 11 cases out of 38 cases with some form of neoplastic involvement. In 9 of these 11 cases, tubal wall invasion or lymphatic involvement was there. So metastatic disease was likely to be the aetiology. In 2 of the 11 cases, only STIC was present and another case showed both STIC and serous carcinoma. All cases of STIC were in patients with stage III or IV disease.

The study conducted by Kiyong $\mathrm{Na}$ et $\mathrm{al}^{9}$ showed that $14(23.3 \%)$ out of 60 tubal metastases were from uterine malignancies. Sherman and Mark et al ${ }^{10}$ studied on 41 uterine papillary serous carcinomas and found out 2 cases (5\%) with fallopian tube changes.

\section{CONCLUSION}

\section{The Conclusions of the Study are as follows}

1. The various histopathological changes in fallopian tube in uterine malignancies were endometriosis, dysplasia, carcinoma, inflammation and salpingitis isthmica nodosa. Endometrioid carcinoma and serous carcinoma of uterus showed more frequent changes in fallopian tube compared to clear cell carcinoma, ESS and carcinosarcoma. 
2. The changes were more seen in fimbrial end compared to middle portion and base of fallopian tube. Thus, adding to the significance of sampling and studying of fimbriae along with other parts of fallopian tube in uterine malignancies.

\section{REFERENCES}

[1] Kulac I, Usubutun A. Microscopic lesions of fallopian tubes in endometrioid carcinoma of the endometrium: How effective are the macroscopic tubal sampling techniques? J Gynaecol Oncol 2013;24(2):114-9.

[2] Carlson J, Roh MH, Chang MC, et al. Recent advances in the understanding of the pathogenesis of serous carcinoma: the concept of low-and high-grade disease and the role of the fallopian tube. Diagnostic Histopathology (Oxf) 2008;14(8):352-65.

[3] Mittal N, Srinivasan R, Gupta N, et al. Secretory cell outgrowths, p53 signatures and serous tubal intraepithelial carcinoma in the fallopian tubes of patients with sporadic pelvic serous carcinoma. Indian Journal of Pathology and Microbiology 2016;59(4):481-8.

[4] Jordan LB, Abdul-Kader M, Al-Nafussi A. Uterine serous papillary carcinoma: histopathological changes within female genital tract. International Journal of Gynaecological Cancer 2001;11(4):283-9.
[5] Alvarado-Cabrero I, Navani SS, Young RH, et al. Tumors of the fimbriated end of the fallopian tube: a clinicopathologic analysis of 20 cases, including nine carcinomas. Int J Gynecol Pathol 1997;16(3):189-96.

[6] Fadare 0, Zheng W, Crispens MA, et al. Morphologic and other clinicopathologic features of endometrial clear cell carcinoma: a comprehensive analysis of 50 rigorously classified cases. American Journal of Cancer Research 2013;3(1):70-95.

[7] Culton LK, Deavers MT, Silva EG, et al. Endometrioid carcinoma simultaneously involving the uterus and the fallopian tube: a clinicopathologic study of 13 cases. Am J Surg Pathol 2006;30(7):844-9.

[8] Tolcher MC, Swisher EM, Medeiros F, et al. Characterization of precursor lesions in the endometrium and fallopian tube epithelium of early stage uterine serous carcinoma. International Journal of Gynecological Pathology 2015;34(1):57-64.

[9] Na K, Kim HS. Clinicopathological characteristics of fallopian tube metastases from primary endometrial, cervical and nongynecological malignancies: a single institutional experience. Virchows Archiv 2017;471(3):363-73.

[10] Sherman ME, Bitterman P, Rosenshein NB, et al. Uterine serous carcinoma. A morphologically diverse neoplasm with unifying clinicopathological features. American Journal of Surgical Pathology 1992;16(6):600-10. 